\title{
Consolidation of Ti-SiC Particle-Reinforced Metal-Matrix Composites
}

\author{
T. Christman, K. Heady and T. Vreeland, Jr. \\ Mail Stop 138-78 \\ Division of Engineering and Applied Science \\ California Institute of Technology \\ Pasadena CA 91125
}

\section{Table of Contents}

1. Introduction .. . . . . . . . . . . . . . . . . . . . . . . . . . 2

2. Ti Alloy Composites . . . . . . . . . . . . . . . . . . . . . . . . . . . . . . . . . 2

3. Ti-SiC Interfaces . . . . . . . . . . . . . . . . . . . . . . . . . . . . . . . . . 3

4. Mechanics of DMMC . . . . . . . . . . . . . . . . . . . . . . . . . . . . . . . 3

5. Shock Consolidation Procedure and Results . . . . . . . . . . . . . . . . . . . . . . . 4

6. Heat Treatment Procedure and Results . . . . . . . . . . . . . . . . . . . . . . . . 6

7. Summary . . . . . . . . . . . . . . . . . . . . . . . . . . . . . . . . . . . . . 6 


\title{
CONSOLIDATION OF Ti-SiC PARTICLE-REINFORCED METAL-MATRIX COMPOSITES
}

\author{
T. Christman, K. Heady and T. Vreeland, Jr. \\ Div. of Eng. \& App. Sci., Cal. Inst. of Tech., Pasadena CA 91125, USA
}

\section{Introduction}

Discontinuously-reinforced metal-matrix composites (DMMC) have received an increasing amount of attention in recent years due to their improved strength, stiffness and wear characteristics. These benefits make DMMC quite appealing for many structural applications; however, limited ductility and toughness have prevented their widespread use. Limited ductility and toughness are not unique to this class of advanced materials and are shared by such systems as continuous fiber composites and monolithic ceramics. The introduction of weak microstructural features in these materials has long been known to enhance the overall fracture properties. These features have taken on many forms depending on the system involved; for example, microcracking grain boundaries in ceramics (1) and weakened interfacial strengths in continuous fiber composites (2). This approach has proven to be quite effective for a wide variety of materials but remains relatively unexplored for DMMC. It has been assumed that the greatest

terfacial strength obtainable will produce an optimized mechanical response. This assumption has not veen challenged to any significant degree mainly because of the experimental inability to effectively vary the interfacial characteristics using standard processing procedures.

The ability to control the evolution of matrix-reinforcement interfaces in DMMC through various processing parameters is of critical importance for both scientific investigations and industrial applications. The extremely short time at the compaction temperature that can be achieved with shock consolidation make it a unique method for the minimization (and subsequent systematic variation) of interfacial reactions. Because of the high reactivity of $\mathrm{Ti}$ with the readily available reinforcement particles, this system possesses significant potential for gain from improved interfacial control. The fundamental questions concerning the "optimum" interfacial strength for a given DMMC can be systematically investigated with these reactive composite materials.

The objective of this study was to produce fully dense DMMC compacts comprised of a reactive particle-matrix combination with little or no interfacial reactions in the as-consolidated condition. This material can provide unique information regarding the role of interfaces in DMMC.

\section{Ti Alloy Composites}

Production of continuous fiber Ti based composites has been feasible for many years and reviews of the state of this technology are available, e.g. (3). The major complication in the development of $\mathrm{Ti}$ matrix composites, compared to other systems, has been the detrimental interfacial characteristics that are often observed with many reinforcement materials. Most of the investigations that deal explicitly with matrix composites are focussed on the characterization and manipulation of matrix-fiber interfacial reactions, e.g. (3-8). The large reaction zone has effectively precluded the processing of DMMC based on $\mathrm{Ti}$ alloys.

A primary practical consideration in the analysis of $\mathrm{Ti}$ based composites is the operating tempera- 
tures to which these materials will be subjected in prospective applications. One potential application is for elevated temperature engine parts where considerable weight savings can be obtained by replacement of superalloy materials with $\mathrm{Ti}$ based composites. These parts would have operating temperatures on Q significant exposure at these temperatures. The importance of investigating not only the as-consolidated interface, but also the microstructure of the composite after thermal exposure is clearly recognized. It should be stated that for these expected working temperatures the Ti-SiC interfaces are stable (see Section 3). The dominant obstacle with respect to detrimental interfacial reactions occurs during the processing of Ti matrix DMMC and is not due to exposure at expected operating temperatures of $600^{\circ}$ C.

\section{Ti-SiC Interfaces}

The reaction products that have been reported for Ti-SiC interfaces depend on the temperature of exposure. At lower temperatures, below about $850^{\circ} \mathrm{C}, \mathrm{TiC}$ is the only observed reaction product (4), whereas, at higher temperatures $\mathrm{Ti}_{5} \mathrm{Si}_{3}$ and limited amounts of ternary Ti-Si-C phases are also formed (5-8). Several investigations into the reaction kinetics of $\mathrm{Ti}$ and $\mathrm{SiC}$ conclude that the reactions are diffusion controlled and suggest several theoretical formulations for the associated reaction zone thickness as a function of time and temperature (5-7). It is important to note that the exposure times associated with these investigations are on the order of hours to hundreds of hours. The processing times encountered in shock consolidation (on the order of microseconds) are not expected to generate significant interfacial reactions.

There would appear to be little doubt that the exposure of the Ti-SiC interfaces to sufficiently elevated temperatures will generate particle-matrix reactions and eventually alter the interfacial strength. At the application temperatures for most $\mathrm{Ti}$ alloys, up to $600^{\circ} \mathrm{C}$, the kinetics are reduced to such an extent that no reaction products are experimentally observed after exposure times on the order of 1000 burs (4). Therefore, it is the consolidation temperatures which have limited the development of Ti-SiC $D M M C$ and not the expected service conditions. These characteristics also make this system ideal for the investigation of the effects of interfacial strength on the far-field mechanical response because the interfacial characteristics can be drastically altered with post-consolidation heat treatments.

\section{Mechanics of DMMC}

Detailed discussions and reviews on the mechanics of particle-reinforced metal-matrix composites can be found in refs. $(9,10)$. Several investigators have attempted to quantify, using finite-element methods, the stress states at the particle matrix interface, the evolution of interfacial voids and the effects on the far-field response of the generation of these voids (11-13). These studies have been based on experimental evidence limited to the investigation of a composite system with a single characteristic interface. The calculations have, in general, been consistent with the limited experimental evidence; however, comparisons with experimental results for composites with variable interfacial strengths have not been performed.

Extensive interfacial void formation is observed in Al-SiC DMMC only during elevated temperature creep tests. Interfacial voids initiate at the corner of the $\mathrm{SiC}$ whisker where the stress concentration is maximum (11-13). The failure is generated by a localization of the strain field and large local tensile hydrostatic stresses. Large compressive hydrostatic stresses along the length of the particle prevents the separation of the interface in regions other than along the far-field loading axis (11). With further straining the particle will then "pullout" from the matrix in a manner similar to that observed in Ontinuous fiber composites. Christman et. al. (12) and Nutt and Duva (13) have published TEM evidence of this mechanism in $\mathrm{SiC}$ reinforced $2124 \mathrm{Al}$ composites. Nutt and Needleman (11) have calculated the alterations in the local stress and strain fields associated with the formation of these voids. 
It should be clearly stated that this type of failure mechanism is predominant only during creep deformation. Other investigators have reported significant particle cracking and ductile matrix failure during quasi-static testing $(9,10)$. Experimental inabilities in generating a failure mode transition (within single composite material and loading pattern) has hindered the assesment of each mode on the far-field nechanical response. Failure mode transitions are possible with a reactive particle-matrix composite.

\section{Shock Consolidation Procedure and Results}

The composites were consolidated with the Keck Laboratory Dynamic Compactor in the Materials Science facility at Caltech. The compactor uses a 3 meter long smooth bore cannon barrel supplied through the courtesy of Aerojet Ordinance. The cannon was designed specifically for the consolidation and recovery of powder materials. A $31.5 \mathrm{~mm}$ diameter flyer plate is mounted into a nylon sabot and accelerated by smokeless shotgun and pistol powder (nitrocellulose). For all the experiments discussed in this report, the flyer plate was a $9 \mathrm{~mm}$ thick disk of 303 stainless steel, having a density of $7.896 \mathrm{gm} / \mathrm{cc}$. Flyer plate velocities are monitored using optical velocimetry techniques. The entire barrel and sample holder are evacuated during the consolidation procedure to a vacuum of $\approx 25 \mathrm{~m}$ Torr.

This facility is capable of producing a nearly plane wave shock front with a minimum of reflected stress waves. The degree of shock wave planarity has been investigated previously with experiments on metallic glass consolidation (14). The control of reflected waves has allowed for the consolidation of composite compacts with few cracks and macroscopic defects. This planarity has also allowed the consolidation of multiple cavity green compacts and near-net-shape composite samples $(14,15)$.

Ti-6Al-4V alloy powder was obtained from Powder Metals Inc. and $\mathrm{SiC}$ powder was obtained from Electro Abrasives Inc. in 2 powder sizes each. The powders were found to be irregular polygons with average sizes given in Table 1 based on optical examinations at 1000x. The standard deviations for the particle dimensions approached $50 \%$ of the given dimension. The $\mathrm{Ti}$ and $\mathrm{SiC}$ powders were mixed asing a medium of petroleum ether at $10 \mathrm{vol} . \% \mathrm{SiC}$. The mixture was shaken vigorously, placed in an ultrasonic bath, and shaken again. The ether was then removed by pouring, heating and ventilation. A measured amount of powder was then placed in the target ring and pressed with 10,000 pounds in the shock direction to form a green compact. The green compact was a disk of $32 \mathrm{~cm}$ diameter and $1.6 \mathrm{~cm}$ in thickness. The distension of the green (theoretical full density/green density) was between 1.9 and 2.0 for all reported cases.

TABLE 1.

Average particle sizes in microns determined by optical microscopy.

\begin{tabular}{|l|c|c|c|}
\hline Powder & Width & Length & Aspect Ratio \\
\hline Ti(small) & 24 & 35 & 1.5 \\
\hline Tillarge) & 80 & 110 & 1.4 \\
\hline SiC(small) & 11 & 15 & 1.4 \\
\hline SiC(large) & 87 & 120 & 1.4 \\
\hline
\end{tabular}

The shock energy deposited in the compact was calculated using standard equations which have been extensively covered in the literature, e.g. (16). For the composite samples, it was assumed that all the deformation in the $\mathrm{SiC}$ particles was elastic and therefore, all the shock energy was deposited into Ge Ti particles. In addition, because of the difficulty in obtaining accurate thermodynamic constants Yor the particular $\mathrm{Ti}$ alloy used, data for elemental $\mathrm{Ti}$ was used in the reported calculations.

Exploratory consolidation attempts were then performed to determine the optimum powder size combinations and shock energies. Shock energies were varied from $190 \mathrm{~J} / \mathrm{gm}$ to $450 \mathrm{~J} / \mathrm{gm}$ over the 
4 possible Ti-SiC powder size combinations. The specific conditions for the consolidation attempts reported here are as follows: flyer plate velocity $\approx 1050 \mathrm{~m} / \mathrm{s}$, shock energy 430 to $450 \mathrm{~J} / \mathrm{gm}$. For all the cases investigated, complete densification was observed by optical and SEM investigations. In addition, excellent $\mathrm{SiC}$ particle distribution was observed in all compacts. Figure 1 shows an optical micrograph typical consolidation.

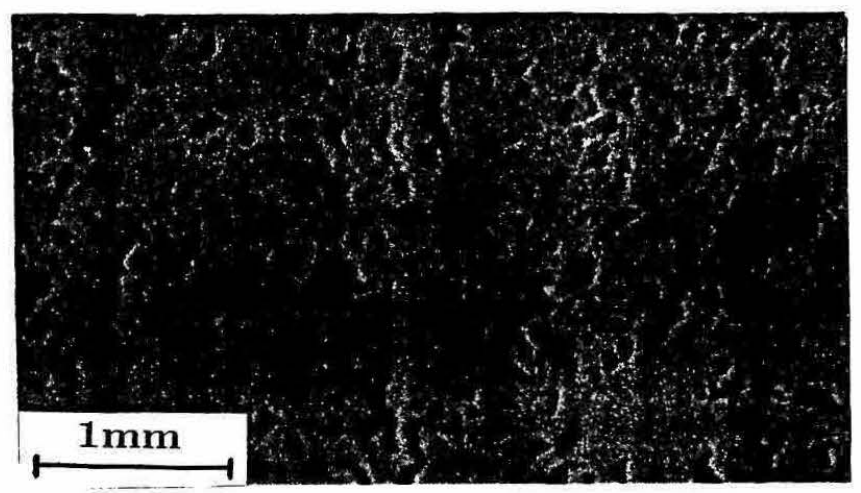

FIG. 1. Optical micrograph of an as-consolidated compact.

The optical and SEM micrographs do not show evidence of interfacial reactions between the Ti matrix and the $\mathrm{SiC}$ particles. In addition, preliminary TEM results on composite samples prepared by ion-milling also do not reveal interfacial reaction zones (17). Therefore, one of the primary goals of this study was achieved, i.e. the production of fully dense Ti-SiC composites with minimal interfacial reactions.

The primary defect observed in the compacts was cracked SiC particles as shown in Figure 2(a) for a compact of large Ti and large SiC powders. Particle cracking was minimized for the case of small 7 nd small $\mathrm{SiC}$ powders, Figure 2(b). Number fractions of cracked versus uncracked particles were determined using optical micrographs and found to range from 0.90 for large Ti and large SiC to 0.05 for small $\mathrm{Ti}$ and small SiC. Variations in the shock energy did not appear to affect the number fraction of cracked $\mathrm{SiC}$ particles. It should be noted that cracked reinforcement particles are present in most DMMC often due to hot extrusion processing.
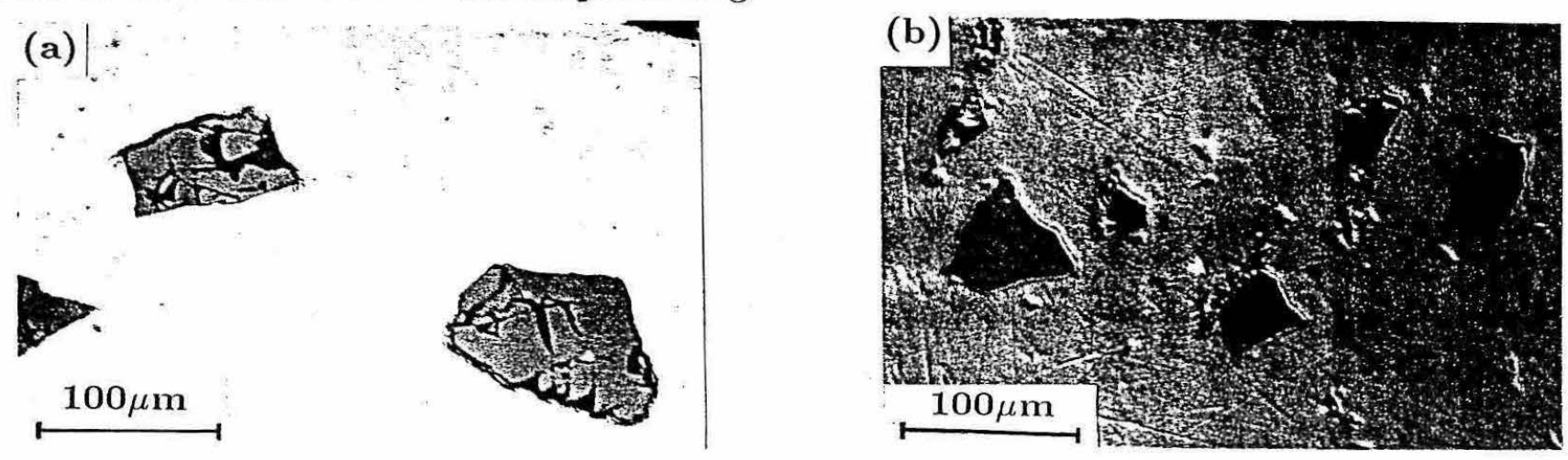

FIG. 2. Micrographs showing the effects of particle size on $\mathrm{SiC}$ particle cracking. Figure 2(a) demonstrates the case of large $\mathrm{Ti}$ and large $\mathrm{SiC}$ and Figure 2(b) demonstrates the case of small $\mathrm{Ti}$ and small $\mathrm{SiC}$.

The processing of near-net shape compacts is also possible using this technique. Figure 3 shows a Ticomposite compact with a complex geometry that was consolidated with zirconia powder surrounding the green compact. Zirconia was chosen because it has similar density and shock wave velocity to that of the composite and would be unlikely to bond to the compact during the shock consolidation. 


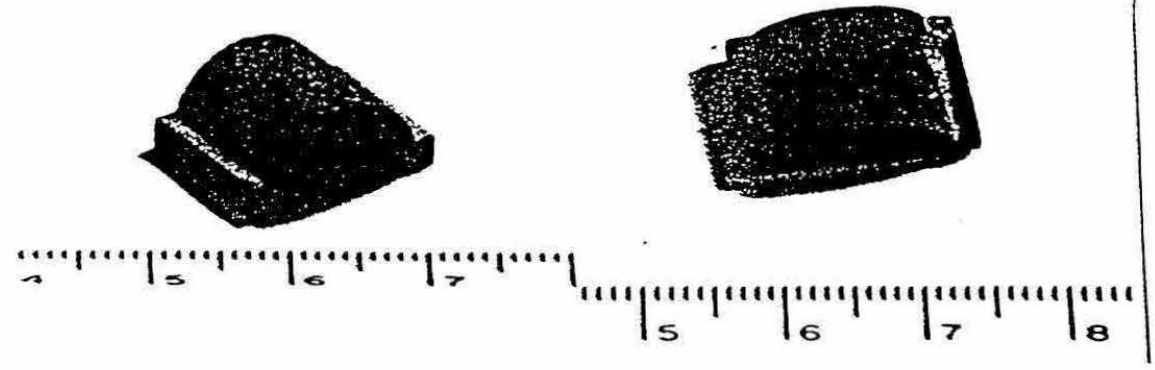

FIG. 3. A near-net shape Ti-SiC composite compact before, $3(\mathrm{a})$, and after, 3(b), consolidation. Note the retention of the sharp corners and flat surfaces. Note also the lack of compression in directions other than that of the shock wave.

\section{Heat Treatment Procedure and Results}

A temperature of $850^{\circ} \mathrm{C}$ is considered standard for annealing Ti-6Al-4V alloys and was chosen as the heat treatment temperature for this reason. As discussed in Section 3, this temperature is sufficient to produce significant reactions between $\mathrm{Ti}$ and $\mathrm{SiC}$. Exposure times of $0.25,1$, 4, and 10 hours were chosen to produce a wide range of interfacial thicknesses. The 0.25 and 1 hour heat treatments were applied in a standard atmosphere furnace and the 4 and 10 hour treatments were applied in an argon atmosphere to prevent significant surface oxidation.

Annealing did not generate interfacial reactions of sufficient extent to be observed by either optical or SEM techniques for the 0.25 and 1 hour heat treatments. Exposures of 4 and 10 hours generated tectable interfacial reaction zones and the results are plotted in Figure 4. Also shown are the predicted reaction zone thicknesses from several investigations. The reactions generated in the present study fall within the range of those predicted from the literature.

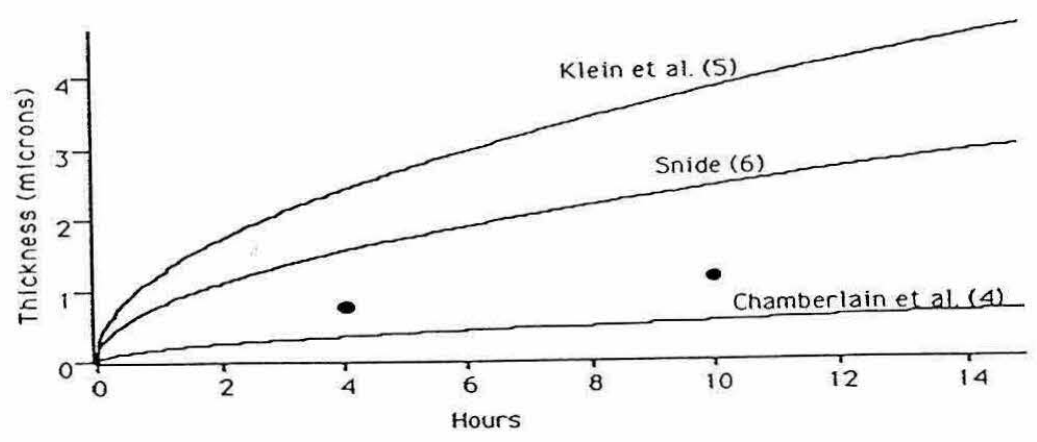

FIG. 4. Calculations of predicted reaction zone thickness from several sources in the literature for exposure times at $850^{\circ} \mathrm{C}$ and the observed thickness from the present investigation (shown by the closed circles).

\section{Summary}

This report details the results of our successful attempt to consolidate a highly reactive particlematrix composite system. This material will be quite useful for the analysis of the effects of interfacial 
reactions on the far-field mechanical response of DMMC. The mechanical characterization of this material is presently underway and will be reported in future publications.

\section{Acknowledgements}

Partial support for this work was provided by the NSF under Grant Number DMR8713258 as a subcontract through The Center for Explosives Technology Research at The New Mexico Institute of Mining and Technology. KH would like to acknowledge the support of the Elmer W. Clark Fellowship at Caltech. The near-net shape consolidation was performed with the able assistance of A. Mutz and B. Krueger.

\section{$\underline{\text { References }}$}

1. M. Ortiz, J. Appl. Mech. 109, 54 (1987).

2. A.G. Metcalf, Interfaces in Metal-Matrix Composites, Vol. 1, Academic Press, New York (1974).

3. P.R. Smith and F.H. Froes, J. Metals, 19 (March 1984).

4. M.B. Chamberlain, Thin Solid Films 72, 305 (1980).

5. M.J. Klein, M.L. Reid and A.G. Metcalf, "Compatibility Studies for Viable Titanium Matrix Composites", AFML Report No. AFML-TR-69-242 (1969).

6. J.A. Snide, "Compatibility of Vapor Deposited B, SiC and $\mathrm{TiB}_{2}$ Filaments With Several Titanium Matricies", AFML Report No. AFML-TR-67-354 (1968).

7. J. Thebault et. al., J. Less Common Metals 47, 221 (1976).

8. J.L. Ratliff and G.W. Powell, "Research on Diffusion in Multiphase Ternary Systems: Reaction Diffusion in the Ti/SiC and Ti-6Al-4V/SiC Systems", AFML Report No. AFML-TR-70-42 (1970).

9. S.V. Nair, J.K. Tien and R.O. Bates, Int. Metall. Rev. 30, 275 (1985).

10. T. Christman, A. Needleman and S. Suresh, Acta Met. 37, 3029 (1989).

11. S.R. Nutt and A. Needleman, Scripta Metall. 21, 705 (1987).

12. S.R. Nutt and J.M. Duva, Scripta Metall. 20, 1055 (1986).

13. T. Christman et. al., Mater. Sci. Engng. 107A, 49 (1989).

14. A. Mutz and T. Vreeland, Jr., "Several Techniques for One-Dimensional Strain Shock Consolidation of Multiple Cavities", in The Proceedings of the International Conference on High Strain-Rate Phenomena in Materials, San Diego, August 12-16, 1990, to be published by Marcel Dekker.

15. B. Krueger and T. Vreeland, Jr., "Shock Induced Reactions in 1:1 Atomic Percent Nickel/Silicon Powder Mixtures", in The Proceedings of the International Conference on High Strain-Rate Phenomena in Materials, San Diego, August 12-16, 1990, to be published by Marcel Dekker.

16. "Dynamic Compaction of Metal and Ceramic Powders", National Materials Advisory Board, NMAB 394, National Academy Press, Washington DC (1983).

17. T. Christman and K. Heady, unpublished research (1990). 